\title{
La mise en défens et la plantation fourragère : deux modes de restauration pour améliorer la végétation, la fertilité et l'état de la surface du sol dans les parcours arides algériens
}

\author{
Fateh Amghar ${ }^{(1)}$, Estelle Langlois ${ }^{(2)}$, Estelle Forey ${ }^{(2)}$, Pierre Margerie ${ }^{(2)}$ \\ (1) Université de Boumerdès. Faculté des Sciences. Département de Biologie. Avenue de l'Indépendance. 35000 Boumerdès \\ (Algérie). E-mail : amgharfateh@univ-boumerdes.dz \\ (2) Université de Rouen. Laboratoire ECODIV EA IRSTEA 1293, FR CNRS 3730 SCALE. Bâtiment IRESE A. Place Émile \\ Blondel. F-76821 Mont Saint Aignan Cedex (France). E-mail : pierre.margerie@univ-rouen.fr
}

Reçu le 27 avril 2015, accepté le 25 janvier 2016.

Description du sujet. Ces quatre dernières décennies ont été marquées par la lutte contre la désertification et la protection des écosystèmes steppiques, devenues des priorités en Algérie. Plusieurs modalités de restauration ont été retenues pour gérer, voire restaurer les parcours steppiques sensibles.

Objectifs. L'étude menée vise à quantifier l'effet de deux techniques de restauration (mise en défens et plantation fourragère) quatre ans après leur mise en place sur le recouvrement par la végétation, les paramètres physico-chimiques du sol et l'état de sa surface dans des parcours arides algériens initialement dégradés.

Méthode. Trois-cent-douze relevés phytoécologiques et pédologiques ont été effectués quatre ans après la mise en œuvre de la gestion (plantation + mise en défens ou mise en défens seule) ainsi que sur des parcelles non aménagées. Des paramètres descripteurs de la végétation, les éléments de la surface du sol et les paramètres chimiques des horizons de surface ont été mesurés pour chaque modalité de traitement.

Résultats. De nombreux paramètres phytoécologiques et édaphiques sont significativement différents dans les espaces restaurés par rapport aux espaces non aménagés, particulièrement les taux d'azote total et de matière organique. Les paramètres granulométriques sont stables.

Conclusions. Notre étude montre que les deux techniques de restauration dans les régions arides permettent en un temps relativement court (quatre ans) une amélioration des propriétés physico-chimiques du sol. Nous suggérons l'utilisation du pourcentage de la surface du sol sous forme d'éléments grossiers comme indicateur de l'état de restauration du système et comme aide à la décision de rouvrir au pâturage les surfaces restaurées.

Mots-clés. Désertification, parcours, plantation, fertilité du sol, propriété de surface du sol, récupération des sols, restauration couverture végétale, Algérie.

Fencing and planting: two restoration strategies for the improvement of vegetation, soil fertility and soil surface properties in Algerian arid rangelands

Description of the subject. The struggle against desertification and the implementation of consecutive steppic ecosystem protection plans have become a priority in Algeria over the last four decades. Among the restoration techniques set up to manage or even restore sensitive steppic rangelands have been (i) fencing and (ii) fencing and the planting of exotic shrubs.

Objectives. This study aims at describing the effect of these two restoration modes with control plots after four years on soil physico-chemical and surface state parameters and phyto-ecological properties.

Method. Three hundred twelve phyto-ecological and pedological relevés were performed both on the restored plots and on controls four years after the initial implementation of management strategies. Phyto-ecological, soil surface and soil chemical parameters were measured for each modality.

Results. Numerous parameters differed between the restored plots and the controls, including levels of total nitrogen and soil organic matter. However, textural parameters did not differ between the restored and the control plots.

Conclusions. Our study shows that the implementation of both the restoration techniques in an arid region led within a relatively short space of time (four years) to an improvement in soil physico-chemical properties. We recommend the measurement of the percentage of coarse elements on the soil surface as an indicator of the system restoration state, and the use of the results as an important decision-making tool in reopening restored areas to grazing.

Keywords. Desertification, rangelands, planting, soil fertility, soil surface properties, reclamation, revegetation, Algeria. 


\section{INTRODUCTION}

Depuis la prise de conscience de l'accélération de l'érosion de la biodiversité par les activités humaines modernes, le manque de connaissances sur les mécanismes régulant les relations entre la biodiversité et le fonctionnement des écosystèmes a été souligné (Grime, 1997) et est devenu un des thèmes centraux en écologie (Wilson, 2000). De la même manière, la dégradation des milieux naturels, suite aux activités anthropiques, suscite depuis plusieurs années des initiatives de restauration en divers endroits dans le monde (UNEP, 1994). Pour cela, les gestionnaires et les scientifiques expriment le besoin d'avoir à leur disposition des concepts et des outils permettant d'optimiser les chances de réussite de leurs projets de restauration.

Les sècheresses du Sahel de 1968-1986 et celles de 1980-1987 en Afrique du Nord ont été le prélude à une vaste prise de conscience des impacts humains sur la biodiversité des systèmes arides et semi-arides et ont suscité un regain d'intérêt pour la compréhension du processus de désertification. Ce processus menace les régions arides et semi-arides (Mainguet, 1990) qui couvrent plus de $35 \%$ des terres émergées (Lal, 2004) et se traduit par une dégradation des milieux associés allant parfois jusqu'à l'irréversibilité du processus sous l'effet combiné des sècheresses récurrentes et d'une exploitation excessive de leurs ressources naturelles entrainant l'installation de conditions de plus en plus défavorables aux êtres vivants (UNEP, 1994 ; Le Floc'h et al., 1995). De nombreux travaux dans le monde ont été consacrés à l'étude de la désertification (Kassas, 1995 ; Puigdefabregas et al., 1998 ; Schonbach et al., 2011 ; Dawelbait et al., 2012), y compris en Algérie avec les travaux de Aidoud (1994) et Slimani et al. (2010). Ces auteurs s'accordent, pour la région considérée, sur une hiérarchie causale impliquant en premier lieu la surexploitation anthropique par le pâturage associée récemment à la mise en culture temporaire massive et en second lieu la sècheresse considérée comme une circonstance aggravante.

La steppe algérienne s'étend sur une superficie de 20 millions d'hectares. Ce territoire constitue un espace vital pour une population estimée en 2008 à presque 12 millions d'habitants. La majeure partie de la population tire ses revenus de la pratique de l'élevage d'un cheptel ovin estimé à plus de 15 millions de têtes. Connue pour la fragilité de son équilibre, la végétation de ces milieux a subi un processus de désertification continu et graduel lié aux pratiques inadaptées de l'homme (surpâturage amplifié par la motorisation du transport du cheptel qui autrefois se déplaçait en suivant les bergers pédestrement). Ces conséquences sont souvent dramatiques pour les ressources naturelles et les populations humaines. Face à cette dégradation du milieu, une stratégie de lutte active contre la désertification a été mise en place pour sauvegarder non seulement la fertilité naturelle des écosystèmes à risque, mais aussi son rétablissement là où cela est techniquement possible. Pour lutter contre cette désertification, l'État a initié dès la fin des années 1960 le barrage vert, plan de plantation forestière d'envergure dans les zones steppiques qui a connu un faible succès (Bensaïd, 1995). En novembre 1994, il a également lancé, sous l'appellation « grands travaux steppiques », des actions pour améliorer le pâturage et la vie rurale. C'est le Haut Commissariat au Développement de la Steppe (HCDS), établissement public à vocation technique et scientifique, qui a eu pour mission la prise en charge des actions en matière de développement intégré des zones steppiques et pastorales. En se basant en partie sur des travaux réalisés dans d'autres pays du Maghreb (plantations d'arbustes tels que Retama retam, mises en exclos), différentes techniques de protection et de restauration des parcours naturels (revégétalisation, mise en défens, travaux hydrauliques, fixation des dunes mobiles, etc.) ont été mises en place sur de très grandes surfaces en Algérie à partir de 1994 (Amiraslani et al., 2011)

En dépit de réticences initiales de la population steppique, il semblerait aujourd'hui que les actions de restauration passive comme la mise en défens (restauration écologique s.s. qui tend à retrouver un ensemble caractéristique d'espèces, de fonctions et de production de restauration comparables à l'écosystème pré-existant ou de référence [SER, 2004]) et active comme la plantation pastorale à base d'Atriplex canescens (qualifiée de réhabilitation au sens de Le Houérou, 2000), engagées par le HCDS, aient rencontré l'adhésion des populations d'agro-pasteurs. Ces actions ont fait l'objet de quelques travaux de suivis dont ceux de Le Houérou (2000) et Amghar et al. (2005) montrant l'amélioration de la richesse floristique et de la valeur fourragère dans les formations restaurées.

Cependant, peu d'études tenant compte des spécificités climatiques, édaphiques et foncières de la steppe algérienne ont été réalisées préalablement à la mise en place massive de programmes de restauration (Bensaïd, 1995) pour évaluer leur efficacité et leur impact à moyen et long terme sur les trajectoires naturelles des communautés végétales. Or, les gestionnaires de ces espaces manifestent de plus en plus leur besoin de données scientifiques solides quant au choix de la technique de restauration à appliquer en fonction du contexte local et de l'état de dégradation.

Le présent travail a pour objectif :

- de caractériser les effets de deux techniques de restauration (la mise en défens et la plantation fourragère à base d'Atriplex canescens), quatre ans après leur mise en œuvre, sur les propriétés physicochimiques du sol, l'état de la surface du sol et les 
descripteurs synthétiques des cortèges floristiques associés ;

- d'identifier parmi ces nombreux paramètres (dont la difficulté et le cout d'acquisition varient) celui ou ceux qui présentent pour les gestionnaires une utilité diagnostique :

- par leur rapidité d'acquisition et leurs faibles cout et technicité,

- par leurs corrélations les plus efficaces et nombreuses possibles avec d'autres variables plus délicates à acquérir,

- par leur capacité à rendre compte de l'état de restauration atteint par le système géré.

\section{MATÉRIEL ET MÉTHODES}

\subsection{Zone d'étude}

L'étude a été menée en Algérie dans la région des hauts plateaux située entre les atlas tellien et saharien dans 17 stations localisées dans 14 communes appartenant à 9 départements steppiques (Figure 1) qui correspondent au domaine steppique nord-africain (Quezel, 1978). Les stations se trouvent entre $32^{\circ} 2$ et $35^{\circ} 3$ de latitude $\mathrm{N}, 1^{\circ} 4 \mathrm{~W}$ et $4^{\circ} 1$ de longitude E, 1000 et $1400 \mathrm{~m}$ d'altitude. Elles sont caractérisées par un bioclimat aride, avec des pluies concentrées en automne et au printemps et un minimum pluviométrique estival correspondant à la période la plus chaude, ce qui fait d'elles des stations à climat méditerranéen (Daget, 1977). Elles présentent une période sèche allant de 6 à 9 mois. En fonction des stations, la moyenne annuelle des précipitations pour la période 1975-2011 oscille entre 230 et $410 \mathrm{~mm}$ et les températures moyennes pour la même période varient respectivement entre $33^{\circ} \mathrm{C}$ et $38^{\circ} \mathrm{C}$ pour les températures maximales du mois le plus chaud et $-0,2{ }^{\circ} \mathrm{C}$ et $5^{\circ} \mathrm{C}$ pour les températures minimales du mois le plus froid. Les sols sont qualifiés de calcimagnésiques xériques à accumulation calcaire qui caractérisent principalement la zone d'étude avec une présence moins importante de sols à encroûtement gypseux (Djebaili, 1978). Ces sols sont colonisés par des groupements végétaux à base de Stipa tenacissima, de Lygeum spartum et d'Artemisia herba alba avec leurs faciès de dégradation (Le Houérou, 1992).

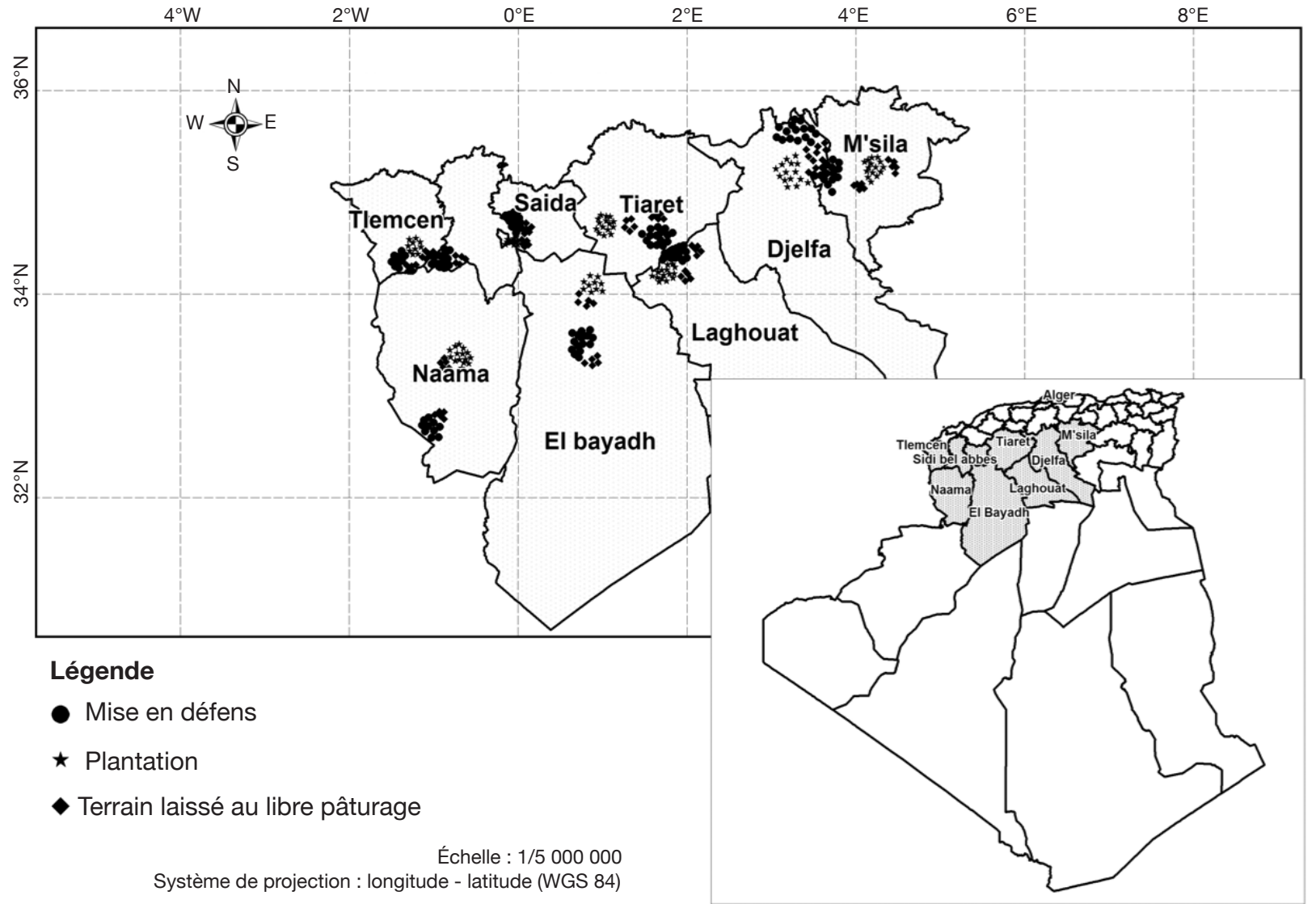

Figure 1. Carte des départements (Wilayas) du nord de l'Algérie montrant les localisations des points d'échantillonnage pour les surfaces non aménagées (= hors mises en défens et hors plantation) ; en encart, la localisation de la zone en Algérie - Map of northern Algeria counties with sampling locations (Hors mise en défens and Hors plantations are control plots). Inset: study area location in Algeria. 


\subsection{Critère du choix du mode de restauration}

Sur la base du critère diagnostique simple qu'est l'état de dégradation de l'unité de gestion au moment où la décision de gestion est prise, l'alternative est la suivante : si la zone est modérément dégradée (pourcentage de couverture végétale pérenne supérieur à $20 \%$ ), la zone est mise en défens. Sa limite est matérialisée par des tumuli visibles de loin et un gardiennage est assuré par 2 à 13 personnes en fonction de la surface. Si la zone est plus dégradée (couverture végétale pérenne inférieure à $20 \%$ ), la zone est également mise en défens, et la gestion par plantation est mise en œuvre en plantant l'arbuste d'origine américaine Atriplex canescens (Chenopodiaceae) élevé pendant un an sous serre à une densité de 1000 plants.ha ${ }^{-1}$. Les plants sont arrosés mensuellement à raison de 101 par plant pendant un an et la zone est mise en défens au même titre que pour la modalité de gestion précédente. L'absence de délimitation physique continue, consécutive à la taille importante des unités de gestion (50 à 400 ha et 1000 à 5000 ha respectivement pour les plantations et les mises en défens), pourrait poser des problèmes de pénétration du cheptel dans ces parcelles. Cette absence est compensée par un mode de choix des parcelles restaurées associant les éleveurs nomades et la structure en charge de la gestion de ces surfaces, le HCDS. Ce mode de décision «bottom up » est un élément important dans le respect des zones plantées ou mises en défens. Les détails de ces modes de restauration ont fait l'objet d'une publication antérieure (Amghar et al., 2012).

\section{3. Échantillonnage}

Pour répondre aux objectifs de cette étude, nous avons adopté une démarche méthodologique basée sur la comparaison des relevés phytoécologiques et pédologiques entre les parcelles restaurées (mise en défens ou plantation) et les parcelles témoins non restaurées situées à proximité 4 ans après la mise en place des modalités de restauration. Dans les parcelles restaurées, les relevés ont été faits dans des plantations ou mises en défens avant la réouverture au pâturage. La courbe aire-espèces (Braun Blanquet et al., 1957) a permis de déterminer une aire minimale de $64 \mathrm{~m}^{2}$. Troiscent-douze placettes de $64 \mathrm{~m}^{2}$ ont été échantillonnées entre 2008 et 2011, afin de faire les relevés dans des formations restaurées depuis la même durée de 4 ans. Le protocole a été mené en partant de l'identification de surfaces floristiquement homogènes (ci-après placettes) dans et au voisinage des parcelles restaurées. Les effectifs de placettes pour chaque catégorie sont les suivants: 122 dans les mises en défens (MD), 105 dans les plantations (P) et 85 dans les surfaces non aménagées $(\mathrm{S})$. La différence d'homogénéité de la végétation dans les parcelles non restaurées par rapport à celles restaurées nous a amenés à faire davantage de placettes de relevés dans les parcelles restaurées. Dans chaque placette, la richesse spécifique $(\mathrm{S})$, le recouvrement de la végétation $(\mathrm{Vg})$, l'indice de Shannon (Shan), l'équitabilité de la végétation (Even), la richesse spécifique en pérennes $(\mathrm{V})$, la richesse spécifique en thérophytes (A) ont été relevés. Les éléments de la surface du sol ont été décrits en suivant la typologie ci-après :

- éléments organiques macroscopiques détachés de la plante mère et en contact avec le sol (nécromasse exclue) : litière ;

- éléments minéraux mobiles :

- éléments minéraux fins consolidés : pellicule de glaçage,

- éléments fins non consolidés : voile éolien ;

- éléments minéraux édaphiques ou géologiques consolidés affleurant suite à l'érosion :

- horizons inférieurs du sol mis en surface et identifiables aux encroûtements cristallins présents, consécutifs à une dénudation : sol nu,

- roche-mère cohésive à granulométrie supérieure aux sables correspondant à un stade d'érosion avancée des matériaux parentaux: éléments grossiers.

Ces éléments ont été quantifiés en suivant la technique du relevé linéaire (Daget et al., 1971). Une ligne de 100 points espacés de $10 \mathrm{~cm}$, dont le point de départ et la direction sont choisis de manière aléatoire dans la surface floristiquement homogène, est tracée puis les espèces végétales et les éléments abiotiques sur ces 100 points sont relevés afin d'estimer leur fréquence qui peut être assimilée à un recouvrement de surface, exprimé en pourcentage (Godron, 1968). La valeur pastorale des placettes a également été calculée sur la base de ces relevés en prenant en compte la valeur fourragère de chaque espèce rencontrée dans les relevés et en la pondérant par son abondance suivant la formule suivante :

$$
V p=C V G \times 0,1 \sum_{i=1}^{R}(C S i \times I s i)
$$

avec $V p$ : valeur pastorale ; $C V G$ : couverture végétale globale du transect ; $R$ : richesse végétale du transect ; $C S i$ : contribution de l'espèce $i$ au recouvrement et Isi : index de qualité spécifique de l'espèce $i$, variant entre 1 et 10, proposé par le CRBT (1978).

Les analyses physico-chimiques ont été réalisées sur l'horizon superficiel H1. Des carottes de $10 \mathrm{~cm}$ ont été prélevées, tamisées à $2 \mathrm{~mm}$ et séchées à l'air libre pour caractériser le sol dans chacune des 312 placettes. Ces mesures sont: le $\mathrm{pH}$ eau et la conductivité électrique déterminés par la méthode du 
$1 / 5$, le taux du calcaire total obtenu par la méthode du calcimètre Bernard (teneur en carbonate par méthode volumétrique suivant la norme ISO 10693), celui du calcaire actif par la Méthode Drouineau-Galet, le taux de la matière organique déterminé par la méthode Anne (carbone organique par l'oxydation sulfo-chromique selon la norme ISO 14235), l'azote total obtenu par la méthode de Kjeldahl (Bremner, 1996). Des analyses granulométriques ont également été réalisées en utilisant la pipette de Robinson.

\subsection{Analyse statistique des données}

La matrice de données de l'ensemble des 312 placettes (312 relevés x 22 descripteurs) a été analysée en utilisant le logiciel $\mathrm{R}$ version 3.1.2 (R Core Team, 2013). L'effet du mode de restauration appliqué par rapport aux surfaces non aménagées sur les éléments de la surface du sol, les paramètres physico-chimiques et ceux de la végétation ont été testés par le test non paramétrique de Kruskal-Wallis suite à la nonnormalité des données (Zar, 1984). La comparaison entre les différents traitements a été effectuée par le test de rang de Wilcoxon. Afin d'identifier les variables les plus synthétiques parmi l'ensemble de celles qui ont été relevées, des corrélations non paramétriques de Kendall sur l'ensemble de la matrice (312 placettes et 22 variables édaphiques et décrivant la végétation) ont été effectuées et testées au seuil de 0,05 par le $\tau$ (tau) de Kendall.

\section{RÉSULTATS ET DISCUSSION}

\subsection{Effet de la gestion sur les paramètres descripteurs de la végétation}

La comparaison des paramètres descripteurs de la végétation des placettes dans les deux modes de restauration et dans les surfaces non aménagées (Tableau 1) confirme les résultats publiés par $\mathrm{Wu}$ et al. (2010) et Amghar et al. (2012). Par rapport aux surfaces non aménagées, la mise en défens présente une richesse spécifique, une richesse en thérophytes et en pérennes et une diversité de Shannon supérieures (Korkanc, 2014 ; Zhang et al., 2015). Les plantations présentent des résultats intermédiaires. Le pourcentage de recouvrement végétal et la valeur pastorale présentent une hiérarchie différente, avec les valeurs les plus élevées pour les plantations et les valeurs intermédiaires pour les mises en défens. Les paramètres à 4 ans peuvent être résumés comme suit : les placettes originellement moins dégradées et mises en défens montrent une trajectoire avec une forte richesse spécifique et une valeur pastorale intermédiaire, alors que les plantations montrent une richesse intermédiaire mais une valeur fourragère élevée. Cet accroissement de la valeur fourragère est principalement dû aux Atriplex plantés.

\subsection{Effet de la gestion sur les états de surface du sol}

En fonction du mode de restauration mis en œuvre (Tableau 1), les pourcentages $\mathrm{du}$ recouvrement végétal, de la litière et de la pellicule de glaçage sont plus importants dans les parcelles restaurées (MD et $\mathrm{P}$ ) alors que ceux du voile éolien, du sol nu et des éléments grossiers augmentent seulement dans les surfaces non aménagées $(\mathrm{S}, p<0,001)$. Plus précisément, le pourcentage de litière est significativement plus élevé dans les mises en défens, intermédiaire dans les plantations et plus faible dans les surfaces non aménagées $(p<0,001)$, ce qui corrobore les résultats de $\mathrm{Su}$ et al. (2004). La production de litière est en relation étroite avec le recouvrement global de la végétation, composée généralement des feuilles et des fragments de feuilles, de fleurs, des organes de fructifications, d'écorce, des fragments de branches et de brindilles, avec une composante de déjections animales et les graines. Dans les milieux pâturés où les conditions sont défavorables (moins d'éléments nutritifs et moins d'humidité), certaines plantes sont capables de libérer dans le milieu par les racines ou leur litière des composés chimiques allélopathiques qui affectent directement la performance des plantes voisines (Carrera et al., 2005), ce qui diminue la richesse floristique de ces milieux. Par ailleurs, le surpâturage affecte négativement les graminées pérennes (surtout Stipa tenacissima et Lygeum spartum), induisant une réduction de la litière qui se traduit par une diminution du carbone organique dans le sol corrélée à la diminution de la $\beta$-glucosidase responsable de la décomposition de la cellulose (Gonzalez-Polo et al., 2009 ; Prieto et al., 2011).

Pour les différents modes de restauration, le développement de la pellicule de glaçage, correspondant ici aux éléments minéraux fins consolidés, montre des différences significatives $(p<0,001)$. Les mises en défens présentent un pourcentage moyen de pellicule de glaçage de 10,09\% contre 2,52\% dans les surfaces non aménagées, alors que sa contribution dans les plantations est de $6,12 \%$. La multiplication par 4 de cette valeur avec la restauration passive peut être consécutive à l'absence du piétinement par le cheptel. Cette pellicule de glaçage joue un rôle important en limitant l'infiltration de l'eau et, augmentant le processus d'érosion, elle peut empêcher l'installation de la végétation et contribue de manière significative à la désertification (Le Houérou, 1992 ; Fox et al., 2004). Selon Aguiar et al. (1997), elle joue un rôle négatif dans le recrutement de nouveaux individus dans les steppes de Patagonie. Étant donné l'effet négatif de 
Tableau 1. Effets des modes de gestion sur les paramètres de la végétation, les états de la surface du sol et les paramètres édaphiques des stations échantillonnées - Management technique effects on vegetation parameters, soil surface states and soil parameters in the sampled stations.

\begin{tabular}{|c|c|c|c|c|c|}
\hline Paramètre ayant trait à la végétation & $S(n=85)$ & $\operatorname{MD}(n=122)$ & $P(n=105)$ & $\chi^{2}$ & Significativité \\
\hline Richesse spécifique & $13,62^{\mathrm{c}}(2,72)$ & $32,30^{\mathrm{a}}(2,25)$ & $24,52^{\mathrm{b}}(2,21)$ & 272,32 & $* * *$ \\
\hline Richesse en annuelles & $5,65^{\mathrm{c}}(1,70)$ & $19,03^{\mathrm{a}}(3,17)$ & $14,84^{\mathrm{b}}(2,99)$ & 223,27 & $* * *$ \\
\hline Richesse en vivaces & $7,98^{\mathrm{c}}(2,72)$ & $13,28^{\mathrm{a}}(2,89)$ & $9,69^{\mathrm{b}}(2,51)$ & 140,23 & $* * *$ \\
\hline Diversité de Shannon & $1,05^{\mathrm{c}}(0,30)$ & $1,43^{\mathrm{a}}(0,35)$ & $1,21^{\mathrm{b}}(0,24)$ & 58,00 & $* * *$ \\
\hline Équitabilité & $0,281^{\mathrm{a}}(0,08)$ & $0,286^{\mathrm{a}}(0,07)$ & $0,263^{\mathrm{b}}(0,05)$ & 8,16 & $*$ \\
\hline Recouvrement végétal (\%) & $27,99^{\mathrm{c}}(6,22)$ & $67,67^{\mathrm{b}}(7,37)$ & $75,25^{\mathrm{a}}(5,87)$ & 214,70 & $* * *$ \\
\hline Valeur pastorale & $10,15^{\mathrm{c}}(3,76)$ & $28,28^{\mathrm{b}}(5,20)$ & $39,10^{\mathrm{a}}(3,74)$ & 259,80 & $* * *$ \\
\hline \multicolumn{6}{|l|}{ État de surface du sol } \\
\hline Litière $(\%)$ & $5,01^{\mathrm{c}}(2,55)$ & $7,98^{\mathrm{a}}(2,29)$ & $6,62^{\mathrm{b}}(2,47)$ & 62,41 & $* * *$ \\
\hline Voile éolien (\%) & $9,28(3,72)$ & $8,81(4,17)$ & $8,52(4,61)$ & 0,55 & ns \\
\hline Pellicule de glaçage $(\%)$ & $2,52 \mathrm{c}(2,42)$ & $10,09^{\mathrm{a}}(3,90)$ & $6,12^{\mathrm{b}}(3,89)$ & 145,46 & $* * *$ \\
\hline Éléments grossiers (\%) & $8,71^{\mathrm{a}}(5,22)$ & $2,19^{\mathrm{b}}(2,99)$ & $1,98^{\mathrm{b}}(3,60)$ & 119,68 & $* * *$ \\
\hline Sol nu $(\%)$ & $47,14^{\mathrm{a}}(9,80)$ & $3,26^{\mathrm{b}}(2,58)$ & $1,60^{\mathrm{c}}(2,21)$ & 203,57 & $* * *$ \\
\hline \multicolumn{6}{|l|}{ Paramètre chimique édaphique } \\
\hline $\mathrm{pH}_{\text {eau }}$ & $7,97^{\mathrm{b}}(0,28)$ & $8,10^{\mathrm{a}}(0,31)$ & $8,12^{\mathrm{a}}(0,28)$ & 11,61 & $* *$ \\
\hline Conductivité $\left(\mathrm{S} \cdot \mathrm{m}^{-1}\right)$ & $0,17^{\mathrm{b}}(0,33)$ & $0,16^{\mathrm{c}}(0,24)$ & $0,26^{\mathrm{a}}(0,30)$ & 51,74 & $* * *$ \\
\hline 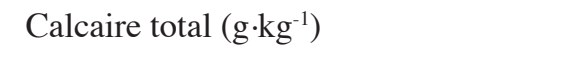 & $8,80^{\mathrm{a}}(8,97)$ & $9,02^{\mathrm{a}, \mathrm{b}}(12,90)$ & $5,67^{\mathrm{b}}(6,52)$ & 10,02 & $* *$ \\
\hline Calcaire actif $\left(\mathrm{g} \cdot \mathrm{kg}^{-1}\right)$ & $5,42^{\mathrm{c}}(4,38)$ & $14,15^{\mathrm{a}}(12,88)$ & $9,78^{\mathrm{b}}(8,81)$ & 25,20 & $* * *$ \\
\hline Matière organique $\left(\mathrm{g} \cdot \mathrm{kg}^{-1}\right)$ & $0,78^{\mathrm{c}}(0,27)$ & $3,06^{\mathrm{b}}(0,63)$ & $3,96^{\mathrm{a}}(0,61)$ & 227,17 & $* * *$ \\
\hline Azote total $\left(\mathrm{g} \cdot \mathrm{kg}^{-1}\right)$ & $0,09^{\mathrm{c}}(0,03)$ & $0,19^{\mathrm{b}}(0,04)$ & $0,24^{\mathrm{a}}(0,06)$ & 186,95 & $* * *$ \\
\hline $\mathrm{C} / \mathrm{N}$ & $4,55^{\mathrm{c}}(0,75)$ & $9,69^{\mathrm{b}}(1,24)$ & $10,04^{\mathrm{a}}(1,60)$ & 184,13 & $* * *$ \\
\hline \multicolumn{6}{|l|}{ Paramètre granulométrique } \\
\hline Argiles (\%) & $9,40(6,76)$ & $9,33(8,84)$ & $7,32(4,75)$ & 3,54 & ns \\
\hline Limons fins (\%) & $6,31(7,58)$ & $6,73(7,32)$ & $7,06(5,94)$ & 4,11 & $\mathrm{~ns}$ \\
\hline Sables $(\%)$ & $84,17(11,13)$ & $83,93(11,43)$ & $85,71(7,79)$ & 0,31 & ns \\
\hline
\end{tabular}

$\mathrm{S}$ : surfaces non aménagées - control ; MD : mises en défens - exclosures ; P : plantations d'Atriplex - Atriplex plantations ; Les valeurs représentent les moyennes et les écarts-type sont entre parenthèses. Les différences sont testées par le test non paramétrique de Kruskal-Wallis $\left(\boldsymbol{\chi}^{2}\right.$ et $p$-value). Les astérisques indiquent le degré de significativité $(* * *: p<0,001, * *: p<0,01 ; *: p<0,05 ; \mathrm{ns}$ : $p>0,05)$. Les différentes lettres indiquent les différences entre les traitements (Kruskal-Wallis rank sum test) - Values are means $\pm S D$. Differences are tested with non parametrical Kruskal-Wallis $\left(\boldsymbol{\chi}^{2}\right.$ and $\mathrm{p}$-value). Significance is indicated by superscripts $(* * *: \mathrm{p}<0,001$; **: $\mathrm{p}<0,01 n s ; *: \mathrm{p}<0,05 ;$ ns: $\mathrm{p}>0,05)$; Different letters indicate differences between the treatments (Kruskal-Wallis rank sum test).

cette pellicule sur l'installation de la végétation par émergence de jeunes plantes (Wallace et al., 1986) et sur l'infiltration de l'eau, nous suggérons qu'une importance particulière soit attachée à ce paramètre lors de l'évaluation de l'effet d'une restauration. Par ailleurs, il est à noter que le taux de matière organique $\mathrm{du}$ sol et l'activité faunistique associée réduisent la surface et la sévérité d'une pellicule de glaçage consécutive à une couverture végétale insuffisante (Dunkerley, 2000).

Dans notre zone d'étude, le sable est omniprésent. Il forme des accumulations diverses, du voile éolien de quelques centimètres d'épaisseur jusqu'aux placages sableux. Le voile éolien, pour les différents aménagements testés, ne présente pas de différence significative. Toutefois, il influence la végétation en conditionnant l'installation d'espèces psammophiles et des thérophytes. Un voile sableux superficiel et discontinu intercepte efficacement l'eau de pluie et favorise l'installation des thérophytes (Amghar et al., 2005). Il ressort que, contrairement au pourcentage d'éléments grossiers et de sol nu, l'ensablement présente des proportions pratiquement identiques entre les placettes restaurées et les placettes de surfaces non 
aménagées avec des valeurs légèrement supérieures pour les milieux non protégés. Ceci peut être mis en relation avec la capacité de la végétation spontanée ou plantée à fixer les sédiments éoliens. Pour les différents modes de restauration, le sol nu présente des différences significatives ( $p$-value $<0,001)$. Les surfaces non aménagées montrent des pourcentages de sol nu plus importants avec en moyenne $47,14 \%$ contre $3,26 \%$ et $1,60 \%$ dans les parcelles restaurées (mise en défens et plantation, respectivement). Ces résultats concordent avec ceux obtenus en 2009 par Schlecht et al. dans les parcours d'Al Jabal al Akhdar dans le nord d'Oman. Le sol nu est dû, selon Yong-Zhong et al. (2005) au piétinement fréquent du cheptel, exposant la surface du sol à l'érosion éolienne. Ceci entraine une diminution de la fertilité du sol et a, par conséquent, un effet négatif sur la végétation (Yates et al., 2000). En outre, le piétinement lié au surpâturage mène au tassement du sol, empêchant l'infiltration de l'eau et donc la germination de graines, réduisant ainsi la couverture végétale et la diversité floristique (Fleischner, 1994). Les pourcentages d'éléments grossiers présentent des différences significatives $(p$-value $<0,001)$. La présence des éléments grossiers est liée à la dégradation de la roche-mère, qui est en relation directe avec l'érosion éolienne et le surpâturage par le piétinement du cheptel. En effet, nous notons en moyenne dans les surfaces non aménagées un pourcentage d'éléments grossiers quatre fois supérieur à celui des milieux aménagés $(p<0,001)$. En 2005, Yong-Zhong et al. ont noté que, en raison du piétinement fréquent par les moutons et les bovins, la surface du sol est devenue dénudée et exposée à l'érosion éolienne et à l'érosion hydrique qui désagrège la croute calcaire. Il en résulte l'exhumation des éléments grossiers et la perte de la fertilité des sols, qui à son tour influence négativement la quantité de végétation qu'ils peuvent soutenir.

\subsection{Effets de la restauration sur les paramètres physico-chimiques édaphiques}

Les résultats des analyses chimiques réalisées sur les carottes prélevées dans les placettes non gérées et des différentes modalités de restauration sont présentés dans le tableau 1. Le $\mathrm{pH}_{\text {eau }}$ est significativement plus élevé dans les plantations et les mises en défens et plus faible dans les surfaces non aménagées $(p<0,01)$. Nos stations sont caractérisées par un $\mathrm{pH}$ neutre à légèrement basique. Le $\mathrm{pH}$ basique dans les milieux restaurés $(8,10 \pm 0,31$ pour les mises en défens et $8,12 \pm 0,28$ dans les plantations) peut être influencé par la litière (Fterich et al., 2012). En effet, dans des plantations d'Atriplex, Zucca et al. (2011) ont noté un pH plus élevé dans 1 'horizon de surface $(0$ à $7,5 \mathrm{~cm})$ sous la canopée d'Atriplex en raison d'une accumulation de sels. Nos résultats concordent avec ceux obtenus par
Zucca et al. (2011), à savoir que le pH augmente avec l'augmentation du carbone organique dans le sol. Dans les milieux pâturés, le $\mathrm{pH}$ est de 7,97 $\pm 0,28$.

La conductivité est significativement plus élevée dans les plantations, intermédiaire dans les surfaces non aménagées et plus faible dans les mises en défens ( $p$-value $<0,001)$. Dans les différents modes de restauration de nos stations, la valeur de la conductivité varie entre $0,16 \mathrm{~S} \cdot \mathrm{m}^{-1}$ et $0,26 \mathrm{~S} \cdot \mathrm{m}^{-1}$, ceci classe nos sols parmi les sols non salés. La conductivité est plus importante dans l'horizon de surface des plantations avec $0,26 \pm 0,30$. Cette augmentation s'expliquerait par la nature de la litière décomposée. Sharma et al. (1973) ont démontré dans leur étude sur les plantations d'Atriplex que l'augmentation de la conductivité est liée aux cations de sodium, potassium, magnésium et calcium libérés par la décomposition des feuilles d'Atriplex et aux échanges importants de sodium entre l'horizon de surface et l'horizon sous-jacent. En effet, les feuilles d'Atriplex canescens renferment par $\mathrm{kg}$ de matière sèche $3 \%$ de sodium, $2,08 \%$ de potassium et $1,3 \%$ de calcium (El Hamrouni et al., $1975^{1}$ cités par Akrimi et al., 1990).

La teneur en calcaire actif est significativement plus élevée dans les mises en défens, intermédiaire dans les plantations et plus faible dans les surfaces non aménagées ( $p$-value $<0,001)$. Nous notons que la teneur en calcaire actif à l'intérieur des deux surfaces restaurées est deux fois supérieure à celle des milieux pâturés, ceci s'expliquerait par une altération plus poussée du calcaire total dans les milieux à meilleure humidité. La présence du calcaire actif dans nos sols suggère que le complexe absorbant est à peu près saturé en calcium avec un $\mathrm{pH}$ compris entre 8 et 8,5 en l'absence d'une quantité suffisante d'humus (Baize, 1988).

Dans les parcours protégés, nous enregistrons un taux de matière organique plus important : 3,06 \pm $0,63 \mathrm{~g} \cdot \mathrm{kg}^{-1}$ pour les mises en défens et $3,96 \pm 0,61 \mathrm{~g}$. $\mathrm{kg}^{-1}$ pour les plantations contre $0,81 \pm 0,74 \mathrm{~g} \cdot \mathrm{kg}^{-1}$ dans les surfaces non aménagées. Les valeurs les plus fortes sont certainement liées à la présence de Stipa tenacissima, Lygeum spartum et Atriplex canescens qui, d'une part, fixent les particules fines du sol chargées en matière organique et, d'autre part, maintiennent l'humidité édaphique favorisant l'activité microbienne et induisant une dégradation de la litière (Prieto et al., 2011). Le recouvrement de la végétation présente un effet positif sur les propriétés du sol (Traoré et al., 2007). Avec l'âge des plantations et des mises en défens, une augmentation du carbone organique est visible avec l'augmentation des résidus

\footnotetext{
${ }^{1}$ El Hamrouni A. \& Sarson M., 1975. Appétabilité de certains Atriplex spontanés ou introduits en Tunisie. Note de recherche $n^{\circ} 8$. Tunis : INRF.
} 
organiques des plantes ainsi que celle des racines qui se décomposent dans le sol (Cao et al., 2008). En effet, l'apport de matière organique via les résidus aériens et souterrains entraine une augmentation de la biomasse microbienne dans le sol, qui se traduit par une meilleure fertilité des sols (Jia et al., 2010). L'âge de la plantation ou de la mise en défens est corrélé positivement à l'activité microbiologique et enzymatique des sols (déshydrogénase, phosphatase et $\beta$-glucosidase), améliorant ainsi leur fertilité (An et al., 2009). Badiane et al. (2001) ont rapporté que les milieux protégés riches en espèces herbacées présentent une meilleure activité enzymatique (surtout la $\beta$-glucosidase) que les milieux pâturés avec une prépondérance de chamaephytes. Par ailleurs, l'augmentation de la matière organique du sol et du stock de nutriments consécutive à la mise en défens pourrait être le résultat d'une augmentation de la quantité de litière végétale d'une part, et d'une diminution de tassement de sol, d'autre part (Xie et al., 2004 ; Yüksek et al., 2011). La diminution importante (division par quatre) de la matière organique entre zones pâturées et zones gérées, outre sa liaison avec la faiblesse du recouvrement végétal, peut être également due au piétinement. En effet, le surpâturage a un effet négatif sur la structure de la végétation, sa composition et diminue la flore tellurique (Fterich et al., 2012) d'une part et, d'autre part, il diminue l'activité biochimique du sol par la dégradation de la structure du sol suite au piétinement (Acosta-Martinez et al., 2010). Selon Fterich et al. (2012), la réduction de l'activité microbienne dans le sol est une réponse aux conditions défavorables résultant du surpâturage (Su et al., 2004 ; Shrestha et al., 2008 ; Steffens et al., 2008).

L'azote total représente le résultat de la minéralisation de la matière organique dans le sol et permet d'apprécier la fertilité du sol (Mazzarino et al., 1998). Sa teneur est significativement plus élevée dans les plantations, intermédiaire dans les mises en défens et plus faible dans les surfaces non aménagées ( $p$-value $<0,001)$. Cette teneur en azote total dans les milieux restaurés est probablement liée à leur soustraction au cheptel permettant le développement des Fabaceae connues pour leur rôle dans la fixation de l'azote atmosphérique, affectant ainsi positivement les propriétés du sol (Traoré et al., 2007). En effet, dans ces milieux, nous avons recensé 27 Fabaceae parmi lesquelles 8 vivaces dont la contribution au tapis végétal se situe entre 15 et $20 \%$ (Argyrolobium uniflorum, Astragalus caprinus, Astragalus gombo, Astragalus tenuifoliosus, Hippocrepis unisiliquosa, Onobrychis alba, Onobrychis argentea et Ononis natrix) et 18 thérophytes parmi lesquelles : Astragalus corrugatus, Hippocrepis ciliata, Medicago laciniata, Ononis serrata, Trigonella monspeliaca et Trigonella polyceratia. Nos résultats rejoignent ceux de Sameni et al. (2007), qui ont montré dans leur étude sur des plantations fourragères d'une région aride de l'Iran que la teneur en azote augmente significativement dans l'horizon de surface $(0-10 \mathrm{~cm})$. En revanche, dans les milieux surpâturés, la faible teneur en azote s'expliquerait par la défoliation qui affecte le stock d'azote dans le sol (Ledgard et al., 1992) d'une part et par la diminution de l'activité des protéases qui affectera sensiblement le potentiel de la minéralisation de l'azote, d'autre part (Su et al., 2004). Prieto et al. (2011) expliquent la diminution du potentiel en azote par l'inhibition de la synthèse des protéases et un changement dans la composition microbienne du sol associée à l'azote des urines déposées par le cheptel. Selon Mazzarino et al. (1996), l'azote est un élément limitant dans les écosystèmes arides ; la diminution de cet élément ainsi que les enzymes telles que les protéases entraineraient un ralentissement de la croissance des végétaux. Dawson et al. (2000) ont démontré l'effet négatif de la défoliation et des urines sur les racines et la flore tellurique. La pauvreté de ces milieux surpâturés en flore tellurique et l'aridité du climat favorisent une minéralisation très lente de la litière.

Les résultats des analyses granulométriques (argiles, limons et sables) ne montrent pas de différences significatives. On peut penser que les paramètres granulométriques des 10 premiers $\mathrm{cm}$ de sol verront leurs valeurs changer significativement à des pas de temps supérieurs aux quatre ans pris comme mesure pour notre étude.

\subsection{Corrélations entre variables et préconisation d'un indicateur de restauration}

La figure 2 montre les corrélations non paramétriques entre les 22 variables relevées dans les 312 placettes lors de cette campagne et testées au seuil de 0,05. Les variables montrant les plus grands nombres de corrélations significatives avec les autres sont: la richesse spécifique (13 variables significativement corrélées), le nombre d'espèces annuelles (12), le rapport $\mathrm{C} / \mathrm{N}$ (11), le pourcentage d'éléments grossiers dans les états de surface du sol (12), celui de sol nu (11).

Dans le cadre d'une mesure routinière d'évaluation de l'état d'une parcelle restaurée, les grandeurs relevant des caractéristiques de la végétation peuvent être complexes et longues à acquérir, alors qu'il n'en va pas de même pour les pourcentages d'état de surface du sol. Les deux mesures des états de surface présentent donc un paramètre plus rapide à acquérir sur le terrain. Il est à noter que le taux d'éléments grossiers est négativement corrélé aux paramètres de végétation suivants : recouvrement global de la végétation $(\mathrm{Vg})$, valeur pastorale $(\mathrm{Vp})$, nombre d'espèces thérophytes (A), richesse spécifique végétale totale (S). Il est 


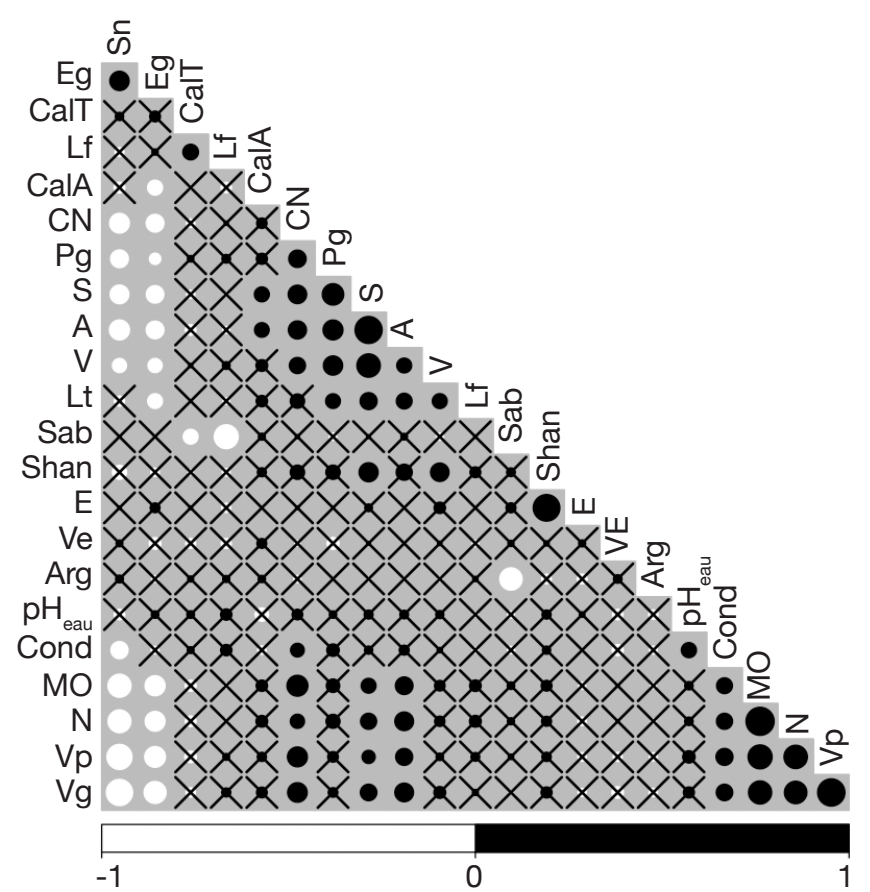

Figure 2. Corrélations non paramétriques entre les 22 variables testées au seuil de 0,05 - Non parametrical correlations between all 22 variables tested at $\mathrm{p}<0.05$.

Les variables sont : pourcentage de sol nu en surface (Sn), pourcentage des éléments grossiers en surface (Eg), Calcaire total (CalT), pourcentage des limons fins granulométriques (Lf), Calcaire actif (CalA), Rapport carbone azote total (CN), pourcentage de pellicule de glaçage en surface $(\mathrm{Pg})$, Richesse spécifique (S), Nombre d'espèces annuelles (A), Nombre d'espèces vivaces $(\mathrm{V})$, pourcentage de litière en surface $(\mathrm{Lt})$, pourcentage de sable granulométrique (Sab), indice de Shannon (Shan), Équitabilité (E), pourcentage de voile éolien en surface (Ve), pourcentage des argiles granulométriques (Arg), $\mathrm{pH}$ (pHeau), Conductivité électrique (Cond), Taux de matière organique (MO), Taux d'azote total $(\mathrm{N})$, Valeur pastorale $(\mathrm{Vp})$, Recouvrement global de la végétation $(\mathrm{Vg})$. Les taux de corrélation de Kendall sont indiqués comme suit : si la $p$-value est $>0,05$, une croix est figurée. Si la $p$-value est $<0,05$, un cercle dont la taille est proportionnelle au taux de corrélation et dont la couleur décrit le sens de la corrélation (négative en blanc, positive en noir) est représenté. Les variables sont ordonnées par une classification ascendante hiérarchique préalable - Variables are: Total vegetation cover (Vg), Organic matter (MO), Pastoral value $(V p)$, Total nitrogen $(N)$, Therophyte species richness $(A)$, Species richness $(S)$, Carbon/Nitrogen ratio $(C N)$, Soil surface sandy crust percentage (Pg), Perennial species richness $(V)$, Conductivity (Cond), Shannon diversity index (Shan), Soil surface litter percentage (Lt), Active lime (CalA), pH (pHeau), Soil granulometric sand percentage (Sab), Soil granulometric fine silt percentage $(L f)$, Evenness $(E)$, Soil surface mobile sand percentage (Ve), Soil granulometric clay percentage (Lf), Soil total lime (CalT), Soil surface coarse elements percentage (Eg), Bare soil surface percentage (Sn). Kendall's correlation rates are plotted as follows : if $\mathrm{p}$-value $>0.05$, the correlation is crossed. If $\mathrm{p}$-value $<0.05$, a circle which size is proportional to correlation rate is figured, colored according to the correlation sense (negative in white, positive in black). Variables are ordined according to an a priori classification. également corrélé aux paramètres de surface suivants : taux de recouvrement par le sol nu (positivement), pellicule de glaçage (négativement), taux de recouvrement par la litière (négativement) et aux paramètres édaphiques suivants: matière organique (négativement), azote total (négativement), rapport $\mathrm{C} / \mathrm{N}$ (négativement), calcaire actif (positivement). Il est à noter que les variables non corrélées au taux d'éléments grossiers sont elles-mêmes corrélées à un faible nombre d'autres variables, par exemple le calcaire total (corrélé à 2 variables), le taux d'argiles (1), les équitabilités (1), le taux des limons fins (2), le taux de sable (2), le pH (1), le taux du voile éolien (0).

Le paramètre «sol nu » montre une amplitude de variation beaucoup plus grande entre les surfaces non aménagées et les placettes restaurées (de $47 \%$ à respectivement 3,26 et 1,60\% pour les mises en défens et les plantations), alors que le paramètre «éléments grossiers » ne présente pas de différence significative entre les mises en défens et les plantations. Cet ensemble de corrélations et la relative facilité de la mesure du paramètre «pourcentage d'éléments grossiers » sur le terrain nous semblent aller dans le sens de sa désignation pour le proposer pour évaluer l'état de réhabilitation ou de restauration d'une parcelle gérée.

\section{CONCLUSIONS}

Quatre ans après la mise en place de modalités de restauration, une amélioration quantitative et qualitative de l'état de la surface du sol est mesurée avec peu de changement pour les paramètres chimiques du sol. La gestion mise en œuvre sur les stations échantillonnées a contribué de manière significative à l'augmentation du pourcentage du recouvrement global de la végétation et de la litière et à une diminution du voile éolien, du sol nu et des éléments grossiers. En revanche, la pellicule de glaçage montre un accroissement important dans les milieux protégés, faute de piétinement. Les travaux consultés s'accordent sur l'effet négatif de cet élément qui est étroitement lié à l'âge de l'aménagement. Dans l'objectif de quantifier rapidement l'effet plus ou moins positif de travaux de restauration sur des surfaces dégradées, nous proposons que le paramètre retenu comme indicateur primaire de l'état de restauration d'une placette soit le pourcentage de sol nu, mais qu'une attention soit apportée au voile éolien pour pondérer la prise de décision de la réouverture d'une formation. Un suivi des paramètres édaphiques et de la végétation après ouverture est nécessaire pour une meilleure compréhension de la désertification et des trajectoires de la végétation des écosystèmes arides et une précision des valeurs seuils qui pourraient être retenues. 


\section{Remerciements}

Les auteurs souhaitent remercier le Haut Commissariat au Développement de la Steppe pour les moyens humains et techniques mis à disposition.

\section{Bibliographie}

Acosta-Martinez V. et al., 2010. Long-term soil microbial community and enzyme activity responses to an integrated cropping-livestock system in a semi-arid region. Agric. Ecosyst. Environ., 137, 231-240.

Aguiar M.R., Paruelo J.M., Sala O.E. \& Lauenroth W.K., 1997. Ecosystem responses to changes in plant functional type composition: an example from Patagonian steppe. J. Veg. Sci., 7, 381-390.

Aidoud A., 1994. Pâturage et désertification des steppes arides en Algérie. Cas de la steppe d'Alfa (Stipa tenacissima L.). Paralelo, 37(16), 33-42.

Akrimi N. \& Zaafouri M.S., 1990. Étude des arbustes fourragers les plus couramment utilisés dans la mise en valeur des régions arides tunisiennes. Descriptionécologie-valeurs pastorales et techniques d'exploitations. Médenine, Tunisie : Institut des Régions Arides, 23-33.

Amghar F., Kadi-Hanifi H. \& Sadji A., 2005. Effect of the fence setting on the pastoral value of five stations of the south Algiers. Option Mediterr. Ser. A, 67, 105-109.

Amghar F. et al., 2012. Grazing exclosure and plantation: a synchronic study of two restoration techniques improving plant community and soil properties in arid degraded steppes (Algeria). Rev. Écol. (Terre Vie), 67(3), 257-269.

Amiraslani F. \& Dragovich D., 2011. Combating desertification in Iran over the last 50 years: an overview of changing approaches. J. Environ. Manage., 92, 1-13.

An S.S., Huang Y.M. \& Zheng F.L., 2009. Evaluation of soil microbial indices along a revegetation chronosequence in grassland soils on the Loess plateau, Northwest China. Appl. Soil Ecol., 41(3), 286-292.

Badiane N.N.Y. et al., 2001. Use of soil enzyme activities to monitor soil quality in natural and improved fallows in semi-arid tropical regions. Appl. Soil Ecol., 18(3), 229238.

Baize D., 1988. Guide des analyses courantes en pédologie. Paris : INRA.

Bensaïd S., 1995. Bilan critique du barrage vert en Algérie. Sécheresse, 6, 247-255.

Braun-Blanquet J. \& De Bolos O., 1957. Les groupements végétaux du bassin moyen de l'Ebre et leur dynamisme. An. Estacion Exp. Aula Dei, 5(1/4).

Bremner J.M., 1996. Nitrogen-Total.In: Sparks D.L.Methods of soil analysis. Part 3. Chemical methods. Madison, WI, USA: Soil Science Society of America, 1085-1122.

Cao C.Y. et al., 2008. Soil chemical and microbiological properties along a chronosequence of Caragana microphylla Lam. plantations in the Horqin sandy land of Northeast China. Appl. Soil Ecol., 40(1), 78-85.
Carrera A.L. et al., 2005. Soil nitrogen in relation to quality and decomposability of plant litter in the Patagonian Monte, Argentina. Plant Ecol., 181, 139-151.

CRBT (Centre de Recherche en Biotechnologie), 1978. Rapport phytoécologique et pastoral sur les hautes plaines steppiques de la wilaya de Saida. Alger : CRBT.

Daget P., 1977. Le bioclimat méditerranéen : caractères généraux, méthodes de classification. Vegetatio, 34, $1-20$.

Daget P. \& Poissonnet J., 1971. Une méthode d'analyse phytosociologique des prairies. Ann. Agron., 22(1), 541.

Dawelbait M. \& Morari F., 2012. Monitoring desertification in a Savannah region in Sudan using Landsat images and spectral mixture analysis. J. Arid Environ., 80, 45-55.

Dawson L.A., Grayston S.J. \& Paterson E., 2000. Effects of grazing on the roots and rhizosphere of grasses. In: Lemaire G., ed. Grassland ecophysiology and grazing ecology. CABI, Cambridge Publishing, 61-84.

Djebaili S., 1978. Recherches phytosociologiques et phytoécologiques sur la végétation des Hautes plaines steppiques et de l'Atlas saharien. Thèse de doctorat: Université Montpellier II Sciences et Techniques du Languedoc (France).

Dunkerley D.L., 2000. Assessing the influence of shrubs and their interspaces on enhancing infiltration in an arid Australian shrubland. Rangeland J., 1, 58-71.

Fleischner T.L., 1994. Ecological costs of livestock grazing in western North-America. Conserv. Biol., 8, 629-644.

Fox D.M., Bryan R.B. \& Price A.G., 2004. The role of soil surface crusting in desertification and strategies to reduce crusting. Environ. Monit. Assess., 99, 149-159.

Fterich A., Mahdhi M. \& Mars M., 2012. Impact of grazing on soil microbial communities along a chronosequence of Acacia tortilis subsp. raddiana in arid soils in Tunisia. Eur. J. Soil Biol., 50, 56-63.

Godron M., 1968. Quelques applications de la notion de fréquence en écologie végétale. Oecologia Plant., 3, 185-212.

Gonzalez-Polo M. \& Austin A.T., 2009. Spatial heterogeneity provides organic matter refuges for soil microbial activity in the Patagonian steppe, Argentina. Soil Biol. Biochem., 41, 1348-1351.

Grime J.P., 1997. Biodiversity and ecosystem function: the debate deepens. Science, 277, 1260-1261.

Jia G.M., Liu B.R., Wang G. \& Zhang B., 2010. The microbial biomass and activity in soil with shrub (Caragana korshinskii K.) plantation in the semi-arid loess plateau in China. Eur. J. Soil Biol., 46, 6-10.

Kassas M.A.F., 1995. Desertification - A general review. J. Arid Environ., 30, 115-128.

Korkanc S.Y., 2014. Effects of afforestation on soil organic carbon and other soil properties. Catena, 123, 62-69.

Lal R., 2004 . Soil carbon sequestration impacts on global climate change and food security. Science, 304, 16231627. 
Le Floc'h E. \& Aronson J., 1995. Écologie de la restauration. Définition de quelques concepts de base. Nat. Sci. Soc., HS, 29-34.

Le Houérou H.N., 1992. An overview of vegetation and land degradation in world arid lands. In: Dregne H.E., ed. Degradation and restoration of arid lands. Lubbock, TX, USA: Texas Tech University, ICASALS, 127-163.

Le Houérou H.N., 2000. Restoration and rehabilitation of arid and semiarid mediterranean ecosystems in North Africa and West Asia: a review. Arid Soil Res. Rehabil., 14, 3-14.

Ledgard S.F. \& Steele K.W., 1992. Biological nitrogen fixation in mixed legume/grass pastures. Plant Soil, 141, 137-153.

Mainguet M., 1990. La désertification : une crise autant socioéconomique que climatique. Sécheresse, 1(3), 187-195.

Mazzarino M.J. et al., 1996. Spatial patterns of nitrogen availability, mineralization, and immobilization in northern Patagonia, Argentina. Arid Soil Res. Rehabil., 10, 295-309.

Mazzarino M.J. et al., 1998. Soil nitrogen dynamics in northeastern Patagonia steppe under different precipitation regimes. Plant Soil, 202, 125-131.

Prieto L.H., Bertiller M.B., Carrera A.L. \& Olivera N.L., 2011. Soil enzyme and microbial activities in a grazing ecosystem of Patagonian Monte, Argentina. Geoderma, 162, 281-287.

Puigdefabregas J. \& Mendizabal T., 1998. Perspectives on desertification: western mediterranean. J. Arid Environ., 39, 209-224.

Quezel P., 1978. Analysis of the flora of Mediterranean and Saharan Africa. Ann. Missouri Bot. Garden, 65(2), 479533.

R Core Team, 2013. R: a language and environment for statistical computing. Vienna: R Foundation for Statistical Computing.

Sameni A.M. \& Soleimani R., 2007. Effect of salinity and some chemical properties of the under- and inter-canopy soils on range plants in a dry region of Southern Iran. Soil Sci. Plant Anal., 38, 15-33.

Schlecht E., Dickhoefer E., Gumpertsberger E. \& Buerkert A., 2009. Grazing itineraries and forage selection of goats in the Al Jabal al Akhdar mountain range of northern Oman. J. Arid Environ., 73, 355-363.

Schönbach P. et al., 2011. Grassland responses to grazing: effects of grazing intensity and management system in an Inner Mongolian steppe ecosystem. Plant Soil, 340(1-2), 103-115.

SER (Society for Ecological Restoration, Science \& Policy Working Group), 2004. SER International Primer on Ecological Restoration, http://www.ser.org/resources/ resources-detail-view/ser-international-primer-onecological-restoration, (26/01/2016).

Sharma M.L.\& Tongway D.J., 1973.Plant induced soil salinity patterns in two saltbush (Atriplex spp.) communities. J. Range Manage., 26, 121-125.

Shrestha G. \& Stahl P.D., 2008. Carbon accumulation and storage in semi-arid sage-brush steppe: effects of long-term grazing exclusion. Agric. Ecosyst. Environ., 125, 173-181.
Slimani H., Aidoud A. \& Rozé F., 2010. 30 years of protection and monitoring of a steppic rangeland under-going desertification. J. Arid Environ., 74, 685-691.

Steffens M., Kolbl A., Totsche K.U. \& Kogel-Knabner I., 2008. Grazing effects on soil chemical and physical properties in semiarid steppe of Inner Mongolia (P.R. China). Geoderma, 143, 63-72.

Su Y.Z., Zhao H.-L., Zhang T.-H. \& Zhao X.-Y., 2004. Soil properties following cultivation and non-grazing of a semiarid sandy grassland in northern China. Soil Tillage Res., 75(1), 27-36.

Traoré S., Thiombiano L., Rasolodimby Millogo J. \& Guinko S., 2007. Carbon and nitrogen enhancement in Cambisols and Vertisols by Acacia spp. in eastern Burkina Faso: relation to soil respiration and microbial biomass. Appl. Soil Ecol., 35, 660-669.

UNEP, 1994. Convention des Nations Unies sur la lutte contre la désertification, http://www.unccd.int/Lists/ SiteDocumentLibrary/conventionText/conv-fre.pdf, (25/01/2016).

Wallace A. \& Wallace G.A., 1986. Effects of soil conditioners on emergence and growth of tomato, cotton and lettuce seedlings. Soil Sci., 141, 313-316.

Wilson S.D., 2000. Heterogeneity, diversity, and scale in plant communities. In: Hutchings M.J., John E.A. \& Stewart A.J., eds. Ecological consequences of habitat heterogeneity. Oxford, UK: Blackwell Science, 53-69.

Wu G.L. et al., 2010. Long-term fencing improved soil properties and soil organic carbon storage in an alpine swamp meadow of western China. Plant Soil, 332(1-2), 331-337.

Xie Y. \& Wittig R., 2004.The impact of grazing intensity on soil characteristics of Stipa grandis and Stipa bungeana steppe in North China (autonomous region of Ningxia). Acta Oecologica, 25, 197-204.

Yates C.J., Norton D.A. \& Hobbs R.J., 2000. Grazing effects on plant cover, soil and microclimate in fragmented woodlands in south-western Australia: implications for restoration. Aust. Ecol., 25, 36-47.

Yong-Zhong S., Yu-Lin L., Jian-Yuan C. \& Wen-Zhi Z., 2005. Influences of continuous grazing and livestock exclusion on soil properties in a degraded sandy grassland, Inner Mongolia, northern China. Catena, 59, 267-278.

Yüksek T. \& Yüksek F., 2011. The effects of restoration on soil properties in degraded land in the semi-arid region of Turkey. Catena, 84, 47-53.

Zar J.H., 1984. Biostatistical analysis. Englewood Cliffs, NJ, USA: Prentice-Hall.

Zhang Y. \& Zhao W., 2015. Vegetation and soil property response of short-time fencing in temperate desert of the Hexi Corridor, northwestern China. Catena, 133, 43-51.

Zucca C., Julitta F. \& Previtali F., 2011. Land restoration by fodder shrubs in a semi-arid agro-pastoral area of Morocco, effects on soils. Catena, 87, 306-312.

(63 réf.) 